\title{
Clinical Application of AR System in Early Rehabilitation Program After Stroke: 2 Case Study
}

\author{
Chang Man An, Dae Hyun Kim \\ Department of Physical Therapy, Chonbuk National University Hospital, Jeonju, Korea
}

Purpose: To investigate the effect of an augmented reality (AR) system on muscle strength and function level of the paretic lower limb and the balance ability in the early rehabilitation program of acute stroke patients.

Methods: The participants (30 or fewer days after stroke) were randomly assigned to receive intervention with an early rehabilitation program using an AR system $(n=1)$ or an early rehabilitation program consisting of functional electrical stimulation and tilt table use $(n=1)$. Patients in both subjects received interventions 4-5 times a week for 3 weeks.

Results: In the paretic limb muscle strength, AR subject was increased from 15 to $39.6 \mathrm{Nm}$ and Control subject was increased from 5 to $30.2 \mathrm{Nm}$. The paretic limb function of AR subject motor function was increased from 8 to 28 score and Control subject motor function was increased from 6 to 14 score. But sensory function was very little difference between the two subjects (AR subject: from 4 to 10 score, Control subject: from 3 to 10 score). In the balance ability, AR subject had more difference after intervention than control subject (AR subject: 33 score, Control subject: 22 score).

Conclusion: The early rehabilitation program using the AR system showed a slightly higher improvement in the motor function of the paretic lower limb and balance ability measurement than the general early rehabilitation program. The AR system, which can provide more active, task-oriented, and motivational environment, may provide a meaningful environment for the initial rehabilitation process after stroke.

Keywords: Augmented reality, Early rehabilitation program, Stroke

\section{INTRODUCTION}

Stroke is a leading cause of movement, sensation, and cognitive impairment, and a diminished ability can persist despite sustained efforts. Eighty-three percent of patients with acute stroke present with postural instability, ${ }^{1}$ and the risk of fall is increased by $73 \%$ in the 6 months following a stroke. ${ }^{2}$ Post-stroke rehabilitation focuses mainly on reducing motor impairment or minimizing physical disability through functional reorganization of the brain. If proper rehabilitation is not achieved early in the disease course, irreversible anatomical or functional changes may occur as well as progressive weakness and disability of the paretic limb. ${ }^{3}$ Traditional rehabilitation therapy programs focus on the needs of individual patients based on assessment findings. However, there is insufficient evidence to suggest

Received Apr 30, 2019 Revised Jun 24, 2019

Accepted Jun 29, 2019

Corresponding author Dae Hyun Kim

E-mail Bicpes4079@gmail.com that these programs are an effective way to improve the function of affected patients. ${ }^{4}$

Virtual rehabilitation recently emerged as a clinical intervention method. This approach is based on virtual reality (VR), augmented reality (AR), and computing technology. This intervention consists of high-intensity training repeats improves athletic performance through a variety of feedback mechanisms, and increases motivation. ${ }^{5,6} \mathrm{AR}$ and VR systems are actively being applied in the poststroke rehabilitation process. AR in particular can enhance authenticity, interactivity, and practicability in a more natural way by providing virtual objects or scenes from the real world provided by the computer. The system also provides automatic visualization for improved posture and motion control by providing biofeedback in real time during the intervention process. ${ }^{7}$ In this process, the pa-

Copylight (C)2019 The Korean Society of Physical Therapy

This is an Open Access article distribute under the terms of the Creative Commons Attribution Non-commercial License (Http:// creativecommons.org/license/by-nc/4.o.) which permits unrestricted non-commercial use, distribution, and reproduction in any medium, provided the original work is properly cited. 
tient is more focused on the movement, which is important to controlling erroneous movements and improving brain plasticity with movements. ${ }^{8,9}$

The functional electrical stimulation (FES) and tilt table are among the most commonly performed interventions for early rehabilitation after stroke. First, FES is applied to contract the paretic lower-limb muscles and induce joint movement. ${ }^{10}$ Pereira et al. ${ }^{11}$ reported that the FES subject had a greater gait walking distance than the gait training subject. Thus, FES increases patient mobility and lower-limb function after stroke. ${ }^{12,13}$ Second, tilt table use reportedly helps with functional recovery by reducing pain, improving bone density, and improving cardiopulmonary function through upright mobilization after stroke. ${ }^{14}$ Repeated training with this device is effective for orthostatic intolerance and spasticity treatment. However, patients tend to be passive when FES or a tilt table is used. FES is used when the patient is seated, while the tilt table is performed with the body fixed to the equipment safely without movement. Therefore, this study applied the AR system to change the passive rehabilitation program environment to a more active patient environment. AR systems provide virtual objects that can be immersed in realworld environments.

The purpose of this AR system is to increase patient interest in performing tasks and help them experience the process of correcting movements through real-time feedback. This can increase patient concentration and motivation and let them experience substantial forces while performing the tasks. Therefore, the purpose of this study was to investigate the effect of the AR system on muscle strength and functional level of the paretic lower limb as well as the balance ability in the initial rehabilitation program of acute stroke patients.

\section{METHODS}

\section{Subject's description}

This study was performed on patients with acute stroke, and the criteria for selection were as follows. Inclusion criteria include 30 days or less after stroke, ${ }^{15}$ currently diagnosed with ischemic or hemorrhagic stroke, adults 18 years or older, Mini-Mental State Examination $>24$, Modified Ashworth Scale $<2$, Berg Balance Test $<20$ (high fall risk), and Those who are admitted to the rehabilitation department ward for 3 weeks or more and can be treated.
Exclusion criteria include past medical history of recurrent syncope, dizziness, visual or hearing impairment, contraversive pushing syndrome or cerebellar lesion, orthopedic injury or diagnosis of the spine or lower extremity, Those diagnosed with heart disease or circulatory system, obesity (body mass index more than $30 \mathrm{~kg} / \mathrm{m}^{2}$ ), If there is a degree of cognitive or communication impairment beyond which this study cannot proceed, Contraindications on tilt tables (cancer, pacemaker, unstable epilepsy). ${ }^{3,16}$ To determine the order of intervention for each subject, randomization (computer-generated) was conducted using a website (http://www.randomization. com). Individual, sequentially numbered index cards with the random assignment were prepared, folded, and placed in sealed opaque envelopes. The AR subjects $(n=1)$ were 57 years old, male, left Hemiplegia due to infarction, duration after stroke: 9, MMSE: 24, BBS: 2. Control subjects $(n=1)$ were 59 years old, left Hemiplegia due to infarction, duration after stroke: 11, MMSE: 24, BBS: 1.

\section{Intervention}

In this study, the early rehabilitation program (a total of three weeks) was divided into FES (30 minutes) and tilt table (30 minutes). The FES applied in the first week was attached to the motor points to stimulate the quadriceps and the tibialis anterior muscle and the peroneal nerve of the paretic side (stimulation of the common peroneal nerve may trigger knee and hip flexion and, thus, the flexion pattern). The frequency of the electrical stimulation was $25-400 \mathrm{~Hz}$.

The tilt table used in the second and third weeks was fixed in the thoracic, pelvic, and bilateral knee through a strap and maintained in the upright position. ${ }^{17}$ During tilt table use, the mediator monitored the clinical observation of dyspnea or pallor. Subjective indices of tolerance for the subjects were: 1) rating of perceived exertion (RPE) 15 as measured by the Borg Rating of Perceived Exertion Scale, ${ }^{18}$ 2) pain using a numerical pain rating ranging from 0 (no pain) to 10 (extreme pain), 3) absence of angina, dizziness, or nausea, and 4) request to discontinue the standing tilt table protocol. ${ }^{19}$

Both subjects also completed daily exercise therapy (30 minutes) related to central nervous system development therapy and limited the therapist to two to limit the deviations associated with exercise therapy (Figure 1).

\section{AR subject}

For this subject, we included the AR system in the early rehabilita- 

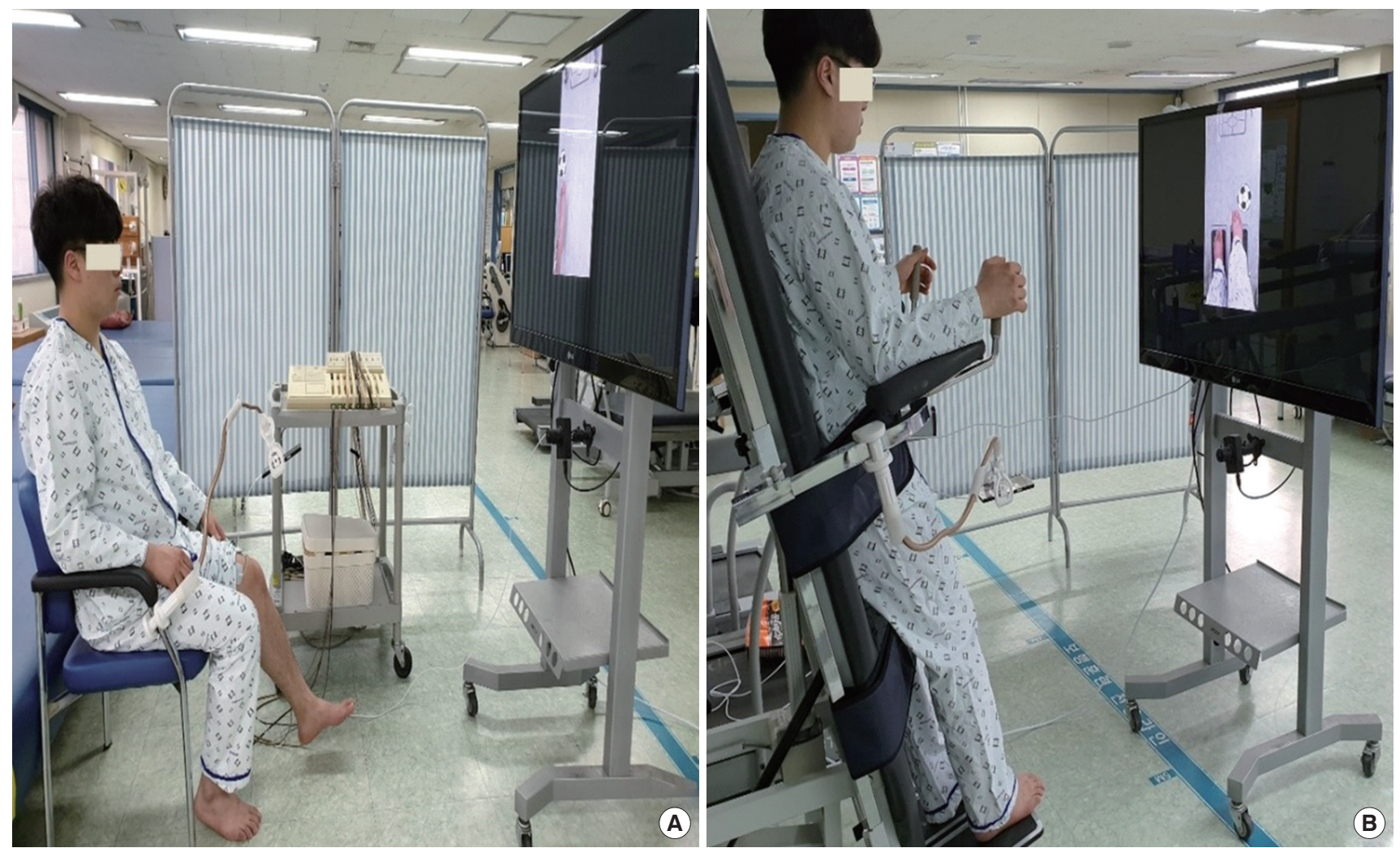

Figure 1. Early rehabilitation program using AR system. (A) Functional electrical stimulation combined AR system, (B) tilt table combined AR system.

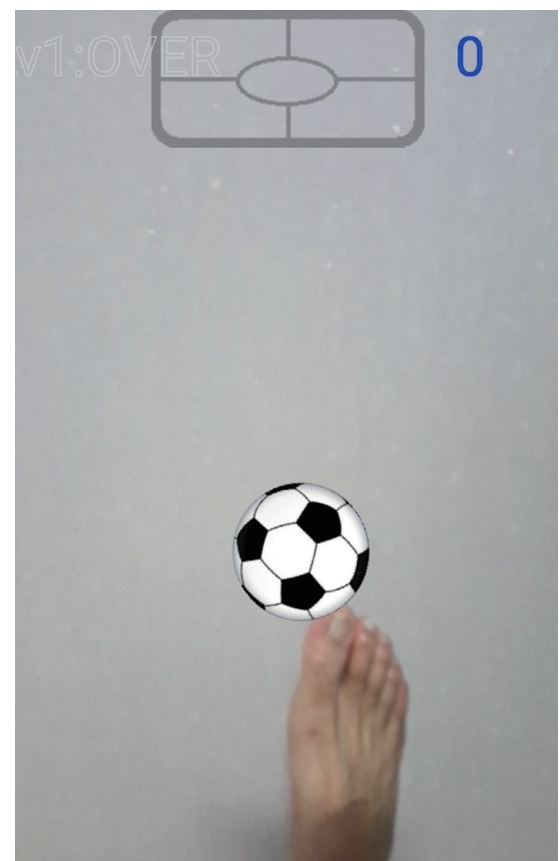

Figure 2. Smart phone application "Kick ball”.

tion program. The AR system consists of a smartphone (Samsung Galaxy note5, Samsung, Korea), TV (LG 55UK6820ENF, LG, Ko- rea), and line (USB to HDMI cable, GNB, China) connecting the two devices to the AR application (Figure 1). The AR application was "Kick Ball (AR soccer, Here you are)," a program that detects one's foot movements to kick the soccer ball on the screen (Figure 2). The subject performed the task of kicking the virtual ball on the monitor toward the goal post. The evaluator constantly monitored the subject during the task and allowed him to immediately take a break in the case of discomfort or fatigue.

During the first week, the subject used the AR system in the course of performing FES while sitting on a chair with a backrest. The subject first played the game using the non-paretic side, followed by the paretic side (15 minutes each). The electrical stimulation intensity of the FES was adjusted so that each subject reached the maximum motion within a tolerable level. The on-off set time of the stimulation was electrically synchronized before the start of the game, when each participant returned the ball to the starting point.

During the second and third weeks, the AR system was applied during the tilt table procedure. At this time, the straps fixed to each site were gradually removed according to the patient's condition. 
This intervention was also performed on the paretic side with the knee area fixed on the strap, followed by the non-paretic side, and then again on the paretic side. The angle of the tilt table was $70^{\circ}-90^{\circ}$ adjusted according to the subject's condition (Figure 2).

\section{Control subject}

For the control subject, the early rehabilitation program was performed without the AR system. The general setting of the FES was the same as that of the AR subject, and the subjects performed knee extension, followed by delivery of the electrical stimulation. The tilt table was used with the application of all straps without any special tasks.

\section{Measurement}

In this study, the isokinetic evaluation system (Biodex System 3PRO, Biodex, USA) was used to evaluate the muscle strength of the quadriceps muscle of the subjects. The subject performs a maximum of knee extensions as strongly as possible, 10 times (2 set), according to the evaluator's instructions. The resting time was given for each set of 10 seconds, and evaluation was performed both on the paretic side and on the non-paretic side. In previous studies, the reliability of this device (ICC) was reported as 0.88 to $0.92 .^{20}$

Fugl-meyer was used to assess the functional (sensory, motor) level of the paretic lower limb of the subject. The Fugl-Meyer scale was designed to quantitatively assess the functional recovery of stroke patients based on the post-stroke recovery stages of Twitchell ${ }^{21}$ and Brunstrom. ${ }^{22,23}$ The total motor function score of the lower limb is 34 points, and the sensory function score is 12 points. It is reported that the lower the score of lower extremity motor function in this evaluation, the more the postural control abnormality increases. This scale has been reported to be useful in evaluating changes in patient's motor function after stroke, ${ }^{24}$ and is highly reliable with interrater reliability $(\mathrm{r}=0.94)$ and intra-rater reliability $(\mathrm{r}=0.99)$.

Balance SD (Biodex Medical Systems, USA) was used to evaluate the subjects' balance ability. The balance indices of this test are reported to reflect well the integrated control of proprioceptive reflexes required to maintain balance and equilibrium. ${ }^{25}$ The limit of stability (LOS) test is a test to evaluate the ability to weight shift the body in eight directions (forward, backward, left, right, forward-left, forward-right, backward-left, and right-backward). When the target points on the screen are blinking and randomly displayed in different directions, the subject moves the center of mass of the body to
Table 1. The comparison of mean scores in before and after intervention

\begin{tabular}{lccc}
\hline Measurement & Baseline & Post-intervention & Difference \\
\hline Muscle strength (Nm) & & 39.6 & 24.6 \\
AR & 15 & 30.2 & 24.8 \\
Control & 5 & & \\
Fugl-Meyer (motor) (score) & & 28 & 20 \\
AR & 8 & 14 & 8 \\
Control & 6 & & \\
Fugl-Meyer (sensory) (score) & & 10 & 6 \\
AR & 4 & 10 & 7 \\
Control & 3 & & \\
Limit of stability (score) & & 33 & 33 \\
AR & NA & 22 & 22 \\
Control & NA & & \\
\hline
\end{tabular}

AR: augmented reality.

reach the target point. The LOS index reflects the trunk imbalance and coordination ability in the lower limb, and the higher the score, the better the ability to balance in a particular direction. ${ }^{24}$ The reliability of this instrument was reported to be as high as the intra-rater reliability $(\mathrm{r}=0.90)$ and inter-rater reliability $(\mathrm{r}=0.94){ }^{26}$

Finally, no specific statistical program was used for data analysis. However, descriptive statistics were applied to the results before and after intervention.

\section{RESULTS}

In the paretic limb muscle strength, AR subject was increased from 15 to $39.60 \mathrm{Nm}$ and Control subject was increased from 5 to $30.20 \mathrm{Nm}$. There was very little difference between the two subjects (Table 1).

The paretic limb function of AR subject motor function was increased from 8 to 28 score and control subject motor function was increased from 6 to 14 score. There was a great difference between the two subjects before and after the intervention. But sensory function was very little difference between the two subjects (AR subject: from 4 to 10 score, Control subject: from 3 to 10 score).

In the balance ability, AR subject had more difference after intervention than control subject (AR subject: 33 score, Control subject: 22 score).

\section{DISCUSSION}

This study aimed to evaluate the effects of intervention with AR 
system on early rehabilitation programs (FES, tilt table) in patients after acute stroke on lower-extremity function, balance ability, and activities of daily living. In the lower-extremity function of the paretic side and dynamic balance ability, AR subject was higher than the control subject for difference score of the before and after intervention. These results suggest that AR systems can improve motor function or skill in stroke patients as reported in previous studies. ${ }^{27-29}$ Bank et al. ${ }^{27}$ and Mousabi et al. ${ }^{30}$ assessed the speed, range, goal-directedness, and smoothness of movements of stroke patients using AR games and reported that this system allows more natural and patient-tailored interactions. Burke et al. ${ }^{28}$ reported that the AR system was better able to improve high-quality motor skills in upper-limb stroke rehabilitation compared with other interventions. Liu et al. ${ }^{29}$ reported that movement skills obtained by training in the AR environment can be transferred to the real-world environment. Thus, studies related to AR systems have mainly focused on the upper-extremity function of stroke patients and have mainly assessed functional level. To date, there is a lack of research on lower-extremity function in stroke patients.

The post-stroke rehabilitation program was reportedly the most important early, intensive, and repetitive factor. ${ }^{31}$ Wade et al. ${ }^{32}$ reported that the early intensive rehabilitation of active functional task produces more positive outcomes. The traditional rehabilitation task is generally uninteresting and boring because of its repetitive nature and the limit obtaining or interpreting data due to the limitation of computer sensing or measurement during the treatment process. ${ }^{33}$ Most therapies are performed on a one-to-one basis in a limited space within the hospital, resulting in high healthcare costs.

The AR system has the advantages of the virtual world and the real world and can provide motivation through virtual objects to provide natural interaction with actual subjects during the rehabilitation process. Virtual environments can safely be used to train functional activities according to individual interests and physical abilities as well as monitor and analyze the subjects' performance data (number of sessions attempted, exercise session length, success rate) over time..$^{34}$ In addition, in the game-designated rehabilitation system, gratifying incentives can promote motivation and enjoyment, creating a greater desire to complete or achieve a specific goal. Burdea $^{33}$ reported that, through the VR rehabilitation system, visual and auditory motivation factors could be provided to alter tradi- tional limited rehabilitation exercises. There are advantages to this system that can reduce the space, cost, or effort required to select physical rehabilitation care protocols.

In this study, FES plays a role in assisting the weak muscular strength of the patients through electric stimulation and providing the timing of the start of movement. When the AR system is used in the tilt table, use of the non-paretic side can have a weight-bearing stimulation effect on the paretic limb, while the use of the paretic side can enhance the strength or function of the ipsilateral lower limb. These tasks focused on the muscle strength or functional movements of the paretic lower limb, which are thought to enable the necessary functional activity transfers in daily life.

There are some limitations to the clinical generalization of the results of this study. First, it is difficult to generalize the outcome of the study to all stroke patients because of the small sample size involved. Second, it is difficult to predict the long-term effects of the intervention because the follow-up evaluation was not performed after the intervention. Third, in the AR application applied in this study, game score was merely a patient motivator, but it is difficult to rate skill improvement or functional enhancement through such scores. Therefore, in future studies, various programs should be developed to clearly score the speed and accuracy of the movement when performing the task, and this score should be interpreted as clinically meaningful.

Finally, this study examined the effect of an AR system in rehabilitation during the acute phase after stroke. The early rehabilitation program using the AR system showed a slightly higher improvement in the motor function of the paretic lower limb and balance ability measurement than the general early rehabilitation program. Based on these results, we propose. The AR system, which can provide more active, task-oriented, and motivational environment, may provide a meaningful environment for the initial rehabilitation process after stroke.

\section{REFERENCES}

1. Tyson SF, Hanley M, Chillala J et al. Balance disability after stroke. Phys Ther. 2006;86(1):30-8.

2. Forster A, Young J. Incidence and consequences of falls due to stroke: a systematic inquiry. BMJ. 1995;311(6997):83-6.

3. Solopova IA, Tihonova DY, Grishin AA et al. Assisted leg displacements and progressive loading by a tilt table combined with FES promote gait recovery in acute stroke. Neuro Rehabilitation. 2011;29(1):67-77. 
4. Puckree T, Naidoo P. Balance and stability-focused exercise program improves stability and balance in patients after acute stroke in a resourcepoor setting. PM and R. 2014;6(12):1081-7.

5. Laver KE, George S, Thomas S et al. Virtual reality for stroke rehabilitation. Cochrane Database Syst Rev. 2011;7(9).

6. Holden MK. Virtual environments for motor rehabilitation: review. Cyberpsychol Behav. 2005;8:187-211.

7. Thikey H, Grealy M, VanWijck F et al. Augmented visual feedback of movement performance to enhance walking recovery after stroke: study protocol for a pilot randomized controlled trial. Trials. 2012;11:13-163.

8. Merians AS, Poizner H, Boian R et al. Sensorimotor training in a virtual reality environment: does it improve functional recovery post stroke? Neurorehabil Neural Repair. 2006;20(2):252-67.

9. Eng K, Siekierka E, Pyk P et al. Interactive visuo-motor therapy system for stroke rehabilitation. Med Biol Eng Comput. 2007;45(9):901-7.

10. Resquín F, Cuesta Gómez A, Gonzalez-Vargas J et al. Hybrid robotic systems for upper limb rehabilitation after stroke: a review. Med Eng Phys. 2016;38(11):1279-88.

11. Pereira S, Mehta S, McIntyre A et al. Functional electrical stimulation for improving gait in persons with chronic stroke. Top Stroke Rehabil. 2012; 19(6):491-8

12. Roche A, Laighin G, Coote S. Surface-applied functional electrical stimulation for orthotic and therapeutic treatment of drop-foot after stroke: a systematic review. Physical Therapy Reviews. 2009;14(2):63-80.

13. Robbins SM, Houghton PE, Woodbury MG et al. The therapeutic effect of functional and transcutaneous electric stimulation on improving gait speed in stroke patients: a meta-analysis. Arch Phys Med Rehabil. 2006; 87(6):853-9.

14. Morris PE. Moving our critically ill patients: mobility barriers and benefits. Crit Care Clin. 2007; 23(1):1-20.

15. Stinear C, Ackerley S, Byblow W. Rehabilitation is initiated early after stroke, but most motor rehabilitation trials are not: a systematic review. Stroke. 2013;44(7):2039-45.

16. Czell D, Schreier R, Rupp R et al. Influence of passive leg movements on blood circulation on the tilt table in healthy adults. J Neuroeng Rehabil. 2004;25(1):4.

17. Zheng X, Chen D, Yan T et al. A randomized clinical trial of a functional electrical stimulation mimic to gait promotes motor recovery and brain remodeling in acute stroke. Behav Neurol. 2018;2018:8923520.

18. Nashner LM. Analysis of movement control in man using the movable platform. Adv Neurol. 1983;39:607-19.

19. Baltz MJ, Lietz HL, Sausser IT et al. Tolerance of a standing tilt table pro- tocol by patients an inpatient stroke unit setting: a pilot study. J Neurol Phys Ther. 2013;37(1):9-13.

20. de Araujo Ribeiro Alvares JB, Rodrigues R, de Azevedo Franke R et al. Inter-machine reliability of the Biodex and Cybex isokinetic dynamometers for knee flexor/extensor isometric, concentric and eccentric tests. Phys Ther Sport. 2015;16(1):59-65.

21. Twitchell TE. The restoration of motor function following hemiplegia in man. Brain. 1951;74(4):443-80.

22. Brunnstrom S. Movement therapy in hemiplegia. New York, Harper \& Row. 1970.

23. Fugl-Meyer AR, Jääskö L, Leyman I et al. The post-stroke hemiplegic patient. 1. a method for evaluation of physical performance. Scand J Rehabil Med. 1975;7(1):13-31.

24. Gladstone DJ, Danells CJ, Black SE. The fugl-meyer assessment of motor recovery after stroke: a critical review of its measurement properties. Neurorehabil Neural Repair. 2002;16(3):232-40.

25. Ganesan M, Pasha SA, Pal PK et al. Direction specific preserved limits of stability in early progressive supranuclear palsy: a dynamic posturographic study. Gait Posture. 2012;35(4):625-9.

26. Cachupe WJC, Shifflett B, Kahanov L et al. Reliability of Biodex balance system measures. Meas Phys Educ Exerc Sci. 2001;5(2):97-108.

27. Bank PJM, Cidota MA, Ouwehand PEW et al. Patient-tailored augmented reality games for assessing upper extremity motor impairments in Parkinson's disease and stroke. J Med Syst. 2018;3;42(12):246.

28. Burke JW, McNeill MDJ, Charles DK et al. Augmented reality games for upper-limb stroke rehabilitation. Proc IEEE Int Conf Games Virtual Worlds Serious Appl. 2010;75-8.

29. Liu J, Mei J, Zhang X et al. Augmented reality-based training system for hand rehabilitation. Multimedia Tools and Applications. 2017;76(13): 14847-67.

30. Mousavi Hondori H, Khademi M, Dodakian L et al. Spatial augmented reality rehab system for post-stroke hand rehabilitation. Stud Health Technol Inform. 2013;184:279-85.

31. Burke JW, McNeill MDJ, Charles DK et al. Optimising engagement for stroke rehabilitation using serious games. Vis Comput. 2009;25:1085-99.

32. Wade DT, Wood VA, Hewer RL. Recovery after stroke the first 3 months. J Neurol Neurosurg Psychiatry. 1985;48(1):7-13.

33. Burdea G. Review paper: Virtual rehabilitation benefits and challenges. Yearb Med Inform. 2003;(1):170-6.

34. Rizzo A, Kim GJ. A SWOT analysis of the field of virtual reality rehabilitation and therapy. Presence. 2005;14(2):119-46. 